\title{
Sleep Duration Modifies the Association of Overtime Work With Risk of Developing Type 2 Diabetes: Japan Epidemiology Collaboration on Occupational Health Study
}

Keisuke Kuwahara ${ }^{1,2}$, Teppei Imai ${ }^{3}$, Toshiaki Miyamoto ${ }^{4}$, Takeshi Kochi ${ }^{5}$, Masafumi Eguchi ${ }^{5}$, Akiko Nishihara ${ }^{3}$, Tohru Nakagawa ${ }^{6}$, Shuichiro Yamamoto ${ }^{6}$, Toru Honda ${ }^{6}$, Isamu Kabe ${ }^{5}$, Tetsuya Mizoue ${ }^{1}$, and Seitaro Dohi ${ }^{7}$, for the Japan Epidemiology Collaboration on Occupational Health Study Group

${ }^{1}$ Department of Epidemiology and Prevention, Bureau of International Health Cooperation, National Center for Global Health and Medicine, Tokyo, Japan

${ }^{2}$ Teikyo University Graduate School of Public Health, Tokyo, Japan

${ }^{3}$ Azbil Corporation, Tokyo, Japan

${ }^{4}$ Nippon Steel \& Sumitomo Metal Corporation Kimitsu Works, Chiba, Japan

${ }^{5}$ Furukawa Electric Co., Ltd., Tokyo, Japan

${ }^{6}$ Hitachi, Ltd., Ibaraki, Japan

${ }^{7}$ Mitsui Chemicals, Inc., Tokyo, Japan

Received September 1, 2016; accepted July 17, 2017; released online February 3, 2018

\begin{abstract}
Background: Evidence linking working hours and the risk of type 2 diabetes mellitus (T2DM) is limited and inconsistent in Asian populations. No study has addressed the combined association of long working hours and sleep deprivation on T2DM risk. We investigated the association of baseline overtime work with T2DM risk and assessed whether sleep duration modified the effect among Japanese.

Methods: Participants were Japanese employees (28,489 men and 4,561 women) aged 30-64 years who reported overtime hours and had no history of diabetes at baseline (mostly in 2008). They were followed up until March 2014. New-onset T2DM was identified using subsequent checkup data, including measurement of fasting/random plasma glucose, glycated hemoglobin, and self-report of medical treatment. Hazard ratios (HRs) of T2DM were estimated using Cox regression analysis. The combined association of sleep duration and working hours was examined in a subgroup of workers $(n=27,590)$.

Results: During a mean follow-up period of 4.5 years, 1,975 adults developed T2DM. Overtime work was not materially associated with T2DM risk. In subgroup analysis, however, long working hours combined with insufficient sleep were associated with a significantly higher risk of T2DM (HR 1.42; 95\% CI, 1.11-1.83), whereas long working hours with sufficient sleep were not (HR 0.99; 95\% CI, 0.88-1.11) compared with the reference ( $<45$ hours of overtime with sufficient sleep).

Conclusions: Sleep duration modified the association of overtime work with the risk of developing T2DM. Further investigations to elucidate the long-term effect of long working hours on glucose metabolism are warranted.
\end{abstract}

Key words: long working hours; sleep habits; Asians; cohort study; prevention

Copyright $\odot 2018$ Keisuke Kuwahara et al. This is an open access article distributed under the terms of Creative Commons Attribution License, which permits unrestricted use, distribution, and reproduction in any medium, provided the original author and source are credited.

\section{INTRODUCTION}

Worldwide, people work for long hours (eg, $\geq 48$ hours per week). ${ }^{1}$ The effect of this on health, especially cardiovascular disease, has been investigated. ${ }^{2}$ Recently, the effect of working long hours on glucose metabolism has gained much attention, although findings are inconsistent. A meta-analysis of cohort studies, mainly from Europe and the United States, ${ }^{3}$ showed no association of working hours with the risk of type 2 diabetes mellitus (T2DM). In Asia, epidemiological evidence is scarce and conflicting. ${ }^{4-7}$

Given that the prevalence of working long hours in Asian countries is higher $(20-50 \%)$ than in European countries
$(10-20 \%),{ }^{1}$ the effect of working long hours on the development of T2DM should be clarified in Asia. Previous Asian studies, however, are limited by relatively small sample sizes (1,000-3,000 participants $)^{4,5,7}$ and the use of simple categorization of working hours into two or three categories. ${ }^{4-6} \mathrm{~A}$ Japanese study reported a higher risk of myocardial infarction among adults with long working hours and short sleep duration, suggesting that sleep may act as an effect modifier. ${ }^{8}$ However, this has not been investigated in terms of the risk of developing T2DM.

We recently reported a cross-sectional association of working hours with having diabetes in a large cohort of Japanese workers. ${ }^{9}$ In the present study, we investigated the prospective association 
between overtime work, including a category of extremely long hours, and risk of T2DM in the same cohort. We also examined the overtime work-T2DM risk association stratified by hours of sleep in a sub-cohort for which the data on sleep were available.

\section{METHODS}

\section{Study procedure}

This cohort study was performed using data on annual health checkups in the sub-cohort of Japan Epidemiology Collaboration on Occupational Health (J-ECOH) Study, ${ }^{10,11}$ an ongoing, multicenter epidemiologic study among workers from more than 10 companies in Japan. Workers in Japan are obliged to have a health checkup at least once per year under the Industrial Safety and Health Act. Before data collection, the conduct of the J-ECOH Study was announced in each of the participating companies using posters to explain the purpose and procedure of the study. The need for participants to provide informed consent for this study was waived. This procedure conforms to the Japanese Ethical Guidelines for Epidemiological Research. The study protocol was approved by the Ethics Committee of the National Center for Global Health and Medicine, Japan.

We extracted data of 52,504 workers (including 8,229 women) at ages 30-64 years who underwent health checkups mainly in 2008 at four companies, and in 2010 at one company, where data on overtime work were available. We followed participants until March 2014.

\section{Participants}

Of the initial 52,504 workers whose data were extracted, we excluded 16,147 at baseline as follows: 7,316 without data on T2DM; 4,074 with pre-existing T2DM; 2,121 who had a history of psychiatric illness $(n=1,024)$, ischemic heart disease $(n=638)$, or cerebrovascular disease $(n=496) ; 4,686$ without data on overtime work; and 5,900 without data on covariates (smoking, $n=5,067$; BMI, $n=84$; and hypertension, $n=1,105$ ). Some participants met more than one of these criteria for exclusion. Lastly, we excluded a further 3,307 workers who did not have any data at subsequent health checkups or who had no data needed to identify T2DM at all subsequent health exams. Thus, data on 33,050 workers (28,489 men and 4,561 women) aged 30-64 years (mean, 44.9; standard deviation [SD], 8.0 years) were included for analysis.

\section{Overtime work hours}

Working hours were measured differently across the four participating companies as described previously ${ }^{9}$ and were integrated for main analysis into four categories from 1 (Short) to 4 (Long). Briefly, in one company, overtime working hours were assessed at each health checkup using a question with response options of: $<45,45-<80,80-<100$, or $\geq 100$ hours per month, and no conversion was made for analysis. A similar question was used in another company: $<45,45-<60,60-<80$, $80-<100$, or $\geq 100$ hours per month in the last $2-3$ months, and the categories of $45-59$ and $60-<80$ hours were integrated into category 2 (second lowest category) for main analysis. In the third company, workers self-reported overtime hours during the 1month period of September, with 11 categories (from " $0-10$ " to ">100 hours" per month), and 0 to 40 hours were converted to the category of 1 (Short), 41-80 hours to 2, 81-100 hours to 3, and $>100$ hours to 4 (Long). In the remaining company, average daily total working hours were self-reported at each health exam. We converted the data on daily working hours into monthly overtime with the formula: (daily working hours -8 ) $\times 20$ days as continuous data and then, classified the data on overtime into four categories (1 to 4$)$.

\section{Biochemical measurements}

Plasma glucose level was estimated using an enzymatic method or a glucose oxidase electrode method. Glycated hemoglobin (HbA1c) level was determined using high performance liquid chromatography method, latex agglutination immunoassay, or an enzymatic method. All laboratories performing these tests received high scores (score $>95$ out of 100 or rank A) by external quality control agencies.

\section{Type 2 diabetes mellitus}

T2DM was diagnosed as a fasting plasma glucose of $\geq 7.0$ $\mathrm{mmol} / \mathrm{L}$, a random plasma glucose of $\geq 11.1 \mathrm{mmol} / \mathrm{L}, \mathrm{HbA} 1 \mathrm{c}$ of $\geq 48 \mathrm{mmol} / \mathrm{mol}$, or current treatment for diabetes. We defined incident cases of T2DM as those who met the diagnostic criteria at any examination after the baseline examination, until March 2014.

\section{Covariates}

Body height and weight were measured based on a standard protocol in each of the participating company. We calculated body mass index (BMI) as weight $(\mathrm{kg})$ divided by the squared height $(\mathrm{m})$. Participants self-reported history of disease and health-related lifestyle factors using a questionnaire, the content of which differed among companies. Hypertension was diagnosed as systolic blood pressure of $\geq 140 \mathrm{~mm} \mathrm{Hg}$, diastolic blood pressure of $\geq 90 \mathrm{~mm} \mathrm{Hg}$, or current treatment for hypertension. Information on working condition, lifestyle habits, and family history of disease was obtained in one company and used for sensitivity analysis to adjust for these variables.

\section{Statistical analysis}

Descriptive data according to overtime hours are shown as mean (SD) for continuous data and number (percentages) for categorical data. Participants were considered to be at risk for T2DM until the date of diagnosis of T2DM or the date of last examination during follow-up, whichever came first. We used Cox regression to calculate hazard ratios (HR) with $95 \%$ confidence intervals (CI) for T2DM. Linear trend was tested by assigning 23, 62, 90, and 120 to categories 1, 2, 3, and 4 of overtime, respectively. Model 1 was adjusted for company (four companies), age (continuous, years), and sex. Model 2 was additionally adjusted for BMI (continuous, $\mathrm{kg} / \mathrm{m}^{2}$ ), smoking (never, past, or current), and factors in model 2 plus HbA1c level (continuous, $\mathrm{mmol} / \mathrm{mol}$ ) at baseline to create Model 3.

In one company, where data on working conditions; lifestyle, including sleep habits; and family history of T2DM were available $(n=27,590)$, we adjusted for sex and age (continuous, year) as Model 4. Alcohol use (non-drinker or drinker consuming $<1,1-2$, and $\geq 2$ go of Japanese sake equivalents a day [1 go of Japanese sake contains approximately $23 \mathrm{~g}$ of ethanol]), smoking status (never, past, or current), occupational physical activity (sedentary, standing or walking, or physically fairly active), department type (field work or non-field work), shift work (yes or no), job position (high or low), family history of T2DM (yes or no), and hypertension (yes or no) at baseline were additionally 
adjusted for Model 5. Sleep duration $(<5.0,5.0-5.9,6.0-6.9$, and $\geq 7$ hours a day) and exercise during leisure $(<2.5$ or $\geq 2.5$ hours a week) at baseline were further adjusted for in Model 6. In Model 7, baseline HbA1c (continuous) was additionally adjusted.

Participants were classified into four groups according to overtime work ( $<45$ or $\geq 45$ hours) and sleep duration $(<5$ or $\geq 5$ hours); the group with $<45$ hours of working overtime and $\geq 5$ hours of sleep was used as the reference group. We examined potential effect modification by sleep on the association of overtime work with T2DM in the fully adjusted model using a likelihood ratio test comparing models with and without interaction terms. All $P$ values are two-sided, and $P$ values $<0.05$ were considered statistically significant. We performed all analyses with Stata statistical software, ver. 14.2 (StataCorp, College Station, TX, USA).

\section{RESULTS}

Baseline characteristics of participants are shown according to overtime work category in Table 1. Participants with longer working hours tended to be male, younger, and had a higher BMI but a lower proportion of hypertension. HbA1c level and smoking prevalence were not materially different according to overtime work category.

During a mean follow-up period of 4.5 years, T2DM occurred in 1,975 participants. In all models, overtime hours were not materially associated with an increase in the risk of T2DM (Table 2). For example, compared with individuals with short overtime work (category 1), the HR of T2DM was 0.94 (95\% CI, 0.64-1.38) for those with long overtime work (category 4) (Model 3, $P$ for trend $=0.97$ ). In the fully adjusted model, sleep duration was associated with T2DM risk in a U-shaped manner $(P$ for quadratic trend $=0.036)$. As compared with sleeping $6-<7$ hours per day, the HR was 1.18 (95\% CI, 0.97-1.45) for sleeping $<5$ hours per day.

Figure 1 shows the combined association of overtime work and sleep duration with T2DM in one company. After adjustment for all covariates, including baseline $\mathrm{HbA1c}$, long overtime ( $\geq 45$ hours) combined with short sleep duration ( $<5$ hours) was associated with a significantly higher risk of T2DM (HR 1.42;

Table 1. Baseline characteristics of participants according to monthly overtime working hours

\begin{tabular}{lllll}
\hline & \multicolumn{4}{l}{ Categories of overtime work (hours per month) } \\
\cline { 2 - 5 } & 1 (Short) & 2 & 3 & 4 (Long) \\
\hline 4 companies & & & & \\
$\quad$ Number of participants & $23,012(69.9)$ & $8,217(25.0)$ & $1,205(3.7)$ & $476(1.5)$ \\
Sex, male & $18,684(81.2)$ & $8,035(97.8)$ & $1,189(98.7)$ & $469(98.5)$ \\
Age, years & $45.6(8.2)$ & $43.4(7.3)$ & $42.9(7.0)$ & $43.3(6.7)$ \\
BMI, kg/m & $23.3(3.3)$ & $23.5(3.1)$ & $23.7(3.1)$ & $23.6(3.1)$ \\
HbA1c, \% & $5.6(0.3)$ & $5.6(0.3)$ & $5.6(0.3)$ & $5.6(0.3)$ \\
Hypertension & $4,156(18.1)$ & $1,046(12.7)$ & $137(11.4)$ & $48(10.1)$ \\
Smoking & $9,115(39.6)$ & $3,246(43.2)$ & $461(38.3)$ & $181(38.0)$ \\
\hline
\end{tabular}

BMI, body mass index; HbAlc, glycated hemoglobin.

Data are shown as mean (SD) for continuous variables and number (percentages) for categorical variables. Overtime working hours were measured differently across the four participating companies and were categorized into the categories of 1 (Short) to 4 (Long). Briefly, in 3 companies, $<45$ hours as category $1,45-<80$ hours as category $2,80-<100$ hours as category 3 , and $\geq 100$ hours as category 4 ; in another company, $<40$ hours as category $1,41-80$ hours as category $2,81-100$ hours as category 3 , and $>100$ hours as category 4 .
95\% CI, 1.11-1.83), whereas long overtime without sleep deprivation was not (HR 0.99; 95\% CI, 0.88-1.11), both compared with monthly overtime of $<45$ hours and $\geq 5$ hours sleep per day $(P$ for interaction $=0.008)$.

\section{DISCUSSION}

We found that overtime work was not associated with increased risk of T2DM. However, long working hours combined with short sleep duration were associated with a significantly higher T2DM risk, whereas individuals with long working hours but without sleep deprivation were not. This is one of the few investigations to address the association of working hours with T2DM incidence in Asia, and the first to report on the effect modification of sleep duration, globally.

Our finding of no association between overtime work and T2DM risk is supported by a meta-analysis using data of cohort studies predominantly in Europe and the United States, ${ }^{3}$ which showed a risk ratio of 1.07 (95\% CI, 0.89-1.27) for working 55 hours per week (approximately 60 hours per month of overtime). In Asia, a cohort study of Japanese civil servants also reported no association with hyperglycemia. ${ }^{6}$ The observed lack of association may be related to the healthy worker effect. Healthy workers, who are at low risk of developing diseases including T2DM, might have worked long hours, resulting in the null finding. In contrast, previous Japanese studies ${ }^{4,5,7}$ showed the opposite findings. Specifically, two Japanese studies reported an inverse association in non-shift workers ${ }^{5}$ and white collar workers ${ }^{7}$ and a positive association in shift workers ${ }^{5}$ and blue collar workers. ${ }^{4}$ Given the inconsistent findings, no confident conclusion can be drawn for the effect of working long hours on glucose metabolism in Asian populations.

We observed that long overtime working hours combined with sleep deprivation showed a higher T2DM risk, whereas long working hours with enough sleep did not. Long working hours may cause sympathetic overactivation, ${ }^{12}$ which leads to hyperglycemia. ${ }^{13}$ In contrast, sufficient sleep is important to inhibit sympathetic activation. ${ }^{14}$ For individuals working long hours, sufficient sleep may be important to recover to a healthy level, whereas insufficient sleep may accentuate the sympathetic overactivation caused by overtime work.

This study has some strengths, including a large sample size, investigation of the effect of extremely long hours of working, and the longitudinal study design. However, limitations should be mentioned. First, working hours were not assessed uniformly across participating companies. Nonetheless, observed associations were not largely different between companies with sufficient numbers of individuals who worked overtime (data not shown). Second, data on working hours and sleep duration were assessed using self-report, so they might be inaccurate to some extent. If random misclassification occurred in these variables, the actual risk associated with overtime work and sleep duration would be higher than observed. Nonetheless, we confirmed that the present questionnaires on overtime or daily working hours are similar to the highly valid and moderately reproducible questionnaires among Japanese employees from the participating companies of J-ECOH Study. ${ }^{15}$ Therefore, the possibility of underestimation would be low. Third, in this study, reference category of overtime work was not no overtime work (0 hours); some participants with short overtime work (eg, $>0$ to $<45$ hours) may have been included in that category. Thus, if short overtime work may 
Table 2. Association of overtime working hours with risk of type 2 diabetes among Japanese workers

\begin{tabular}{|c|c|c|c|c|c|}
\hline & \multicolumn{4}{|c|}{ Categories of overtime work (hours per month) } & \multirow{2}{*}{$P$ for trend ${ }^{\mathrm{a}}$} \\
\hline & 1 (Short) & 2 & 3 & 4 (Long) & \\
\hline \multicolumn{6}{|l|}{ Four companies } \\
\hline Number of cases & 1,362 & 472 & 71 & 27 & \\
\hline Number of subjects & 23,012 & 8,217 & 1,205 & 476 & \\
\hline Person-years & 101,777 & 38,383 & 5,627 & 2,189 & \\
\hline Model $1^{\mathrm{b}}$ & 1.00 (reference) & $0.95(0.86,1.06)$ & $0.95(0.75,1.20)$ & $0.91(0.62,1.33)$ & 0.33 \\
\hline Model $2^{\mathrm{c}}$ & 1.00 (reference) & $1.00(0.90,1.11)$ & $1.03(0.81,1.31)$ & $0.99(0.67,1.45)$ & 0.95 \\
\hline Model $3^{\mathrm{d}}$ & 1.00 (reference) & $0.99(0.88,1.10)$ & $1.07(0.85,1.36)$ & $0.94(0.64,1.38)$ & 0.97 \\
\hline \multicolumn{6}{|l|}{ One company ${ }^{\mathrm{e}}$} \\
\hline Number of cases & 1,092 & 461 & 60 & 23 & \\
\hline Number of subjects & 18,265 & 7,837 & 1,010 & 410 & \\
\hline Person-years & 82,857 & 36,763 & 4,749 & 1,930 & \\
\hline Model $4^{\mathrm{f}}$ & 1.00 (reference) & $0.96(0.86,1.08)$ & $1.00(0.77,1.29)$ & $0.94(0.62,1.42)$ & 0.57 \\
\hline Model $5^{\mathrm{g}}$ & 1.00 (reference) & $1.05(0.94,1.18)$ & $1.17(0.90,1.52)$ & $1.07(0.71,1.62)$ & 0.22 \\
\hline Model $6^{\mathrm{h}}$ & 1.00 (reference) & $1.04(0.93,1.17)$ & $1.13(0.87,1.48)$ & $1.02(0.67,1.55)$ & 0.38 \\
\hline Model $7^{\mathrm{i}}$ & 1.00 (reference) & $1.02(0.91,1.14)$ & $1.15(0.88,1.50)$ & $0.94(0.61,1.43)$ & 0.64 \\
\hline
\end{tabular}

Data are shown as hazard ratio (95\% confidence intervals).

${ }^{a} P$ for trend was calculated by assigning 23, 62, 90, and 120 to increasing categories of overtime work with treating this variable as continuous one.

${ }^{\mathrm{b}}$ Adjusted for age (years, continuous), sex, and worksite (4 companies).

${ }^{\mathrm{c}}$ Additionally adjusted for smoking (never, past, or current), body mass index ( $\mathrm{kg} / \mathrm{m}^{2}$, continuous), and hypertension (yes or no).

${ }^{\mathrm{d}}$ Further adjusted for HbA1c (\%, continuous).

${ }^{\mathrm{e} O v e r t i m e}$ work was measured using a question with 5 response options: $<45,45-<60,60-<80,80-<100$, and $\geq 100$ hours per month.

${ }^{\mathrm{f}}$ Adjusted for age (continuous, year) and sex.

${ }^{g}$ Adjusted for baseline factors including age, sex, smoking (never, past, or current), alcohol consumption (non-drinker or drinker consuming $<1,1-2$, and $\geq 2$ go of Japanese sake equivalents per day), occupational physical activity (sedentary, standing or walking, and physically fairly active), shift work (yes/no), type of department (field-work related or not), job position (high/low), family history of diabetes (yes/no), and hypertension (yes/no).

${ }^{\mathrm{h}}$ Adjusted for factors in model 6 plus leisure-time exercise $(<150$ or $\geq 150$ min per week) and sleep duration $(<5.0,5.0-5.9,6.0-6.9$, and $\geq 7$ hours per day) at baseline.

${ }^{\mathrm{i}}$ Further adjusted for baseline $\mathrm{HbA1c}$ (continuous, mmol/mol).

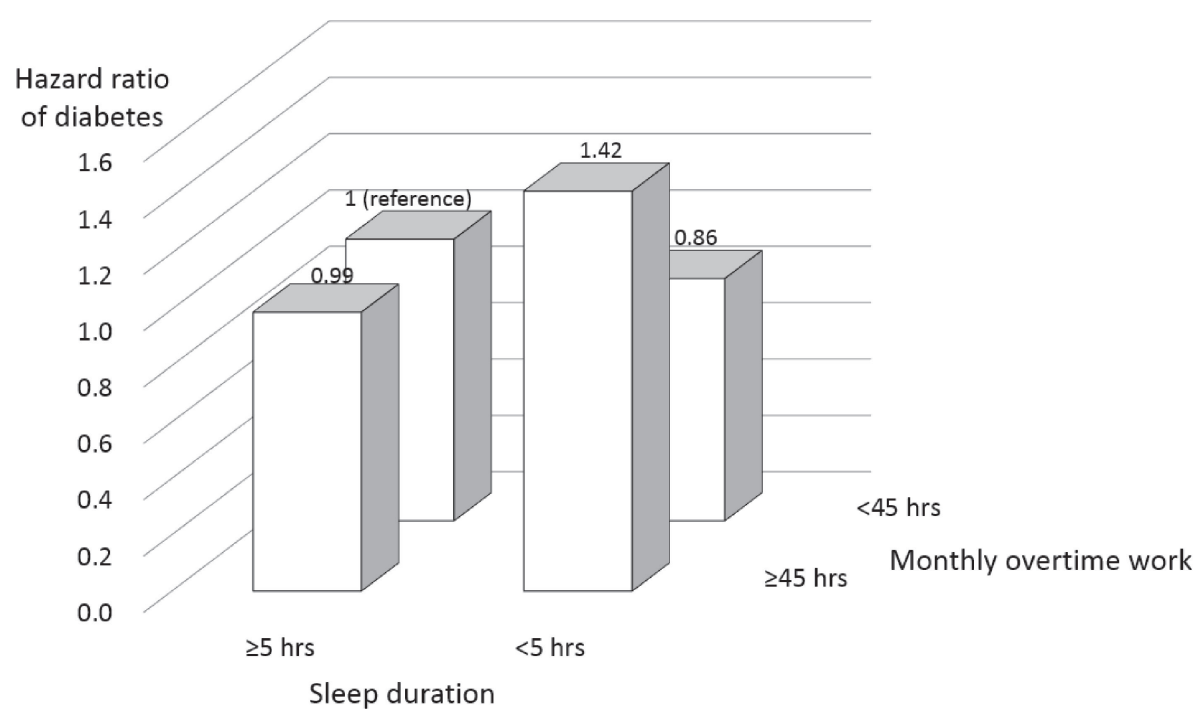

Figure 1. Combined association of overtime work and sleep duration with risk of type 2 diabetes. Data were adjusted for baseline variables, including age, sex, smoking, alcohol consumption, leisure-time exercise, occupational physical activity, shift work, job position, type of department, family history of diabetes, hypertension, body mass index, and HbA1c. HbA1c, glycated hemoglobin.

elevate diabetes risk, the risk associated with long overtime work might have been underestimated. Fourth, only baseline data were used for overtime work. Random changes in working hours during follow-up might have skewed the results toward the null. Fifth, in the participating companies, retirement age was generally set as 60 years, and those who retired at age 60 years may be rehired up to the age 65 years. Thus, workers aged 60 years or older at the entry were excluded mainly due to no follow- up data, potentially leading to biased results. However, exclusion of workers aged 60 years $(n=875)$ did not change the findings (data not shown). Sixth, unmeasured confounders, including socioeconomic status, and residual confounding might have affected the results. Nonetheless, in one company, adjustment for wide array of potential confounders did not change the findings substantially. Lastly, the participants worked in large-scale companies; the present findings may not be applicable to workers 
in companies with different background, including small- to medium-sized companies.

This study of Japanese workers from large-scale companies revealed that overtime work was not associated with an increase in T2DM risk. However, long overtime work was associated with an increased risk of T2DM among those who slept short hours. Further investigations are needed to clarify the long-term effect of long working hours on glucose metabolism and the modification of this effect by sleep deprivation.

\section{ACKNOWLEDGEMENTS}

The authors thank Toshiteru Ohkubo (Chairperson of the Industrial Health Foundation, Japan) for scientific support on the J-ECOH Study; Maki Konishi (National Center for Global Health and Medicine) for management of data; and Rika Osawa (National Center for Global Health and Medicine) for administrative support.

This study was funded in part by the Industrial Health Foundation, JSPS KAKENHI Grant Number 25293146 and 16K21379, the Industrial Disease Clinical Research Grants (150903-01), and the Grant of National Center for Global Health and Medicine (28-Shi-1206). The funders had no role in the present study.

Teppei Imai and Akiko Nishihara belong to Azbil Corporation; Toshiaki Miyamoto, Nippon Steel \& Sumitomo Metal Corporation Kimitsu Works; Takeshi Kochi, Masafumi Eguchi, and Isamu Kabe, Furukawa Electric Co., Ltd.; Tohru Nakagawa, Toru Honda, and Shuichiro Yamamoto, Hitachi, ltd.; and Seitaro Dohi, Mitsui Chemicals, Inc. Teppei Imai, Akiko Nishihara, Toshiaki Miyamoto, Takeshi Kochi, Masafumi Eguchi, Isamu Kabe, Tohru Nakagawa, Toru Honda, Shuichiro Yamamoto, and Seitaro Dohi are occupational physicians in each of the participating companies.

Conflicts of interest: TI, T Miyamoto, TK, ME, AN, TN, SY, $\mathrm{TH}$, IK are health professionals in the participating companies. All authors declare no conflict of interest.

\section{REFERENCES}

1. Lee S, McCann D, Messenger J. Working Time Around the World. Trends in working hours, laws and policies in a global comparative perspective. London: Routledge; 2007.

2. Bannai A, Tamakoshi A. The association between long working hours and health: a systematic review of epidemiological evidence. Scand J Work Environ Health. 2014;40:5-18.
3. Kivimäki M, Virtanen M, Kawachi I, et al. Long working hours, socioeconomic status, and the risk of incident type 2 diabetes: a meta-analysis of published and unpublished data from 222120 individuals. Lancet Diabetes Endocrinol. 2015;3:27-34.

4. Kawakami N, Araki S, Takatsuka N, Shimizu H, Ishibashi H Overtime, psychosocial working conditions, and occurrence of noninsulin dependent diabetes mellitus in Japanese men. J Epidemiol Community Health. 1999;53:359-363.

5. Bannai A, Yoshioka E, Saijo Y, Sasaki S, Kishi R, Tamakoshi A The risk of developing diabetes in association with long working hours differs by shift work schedules. J Epidemiol. 2016;26:481487

6. Itani O, Kaneita Y, Ikeda M, Kondo S, Murata A, Ohida T. Associations of work hours and actual availability of weekly rest days with cardiovascular risk factors. J Occup Health. 2013;55:1120.

7. Nakanishi N, Nishina K, Yoshida H, et al. Hours of work and the risk of developing impaired fasting glucose or type 2 diabetes mellitus in Japanese male office workers. Occup Environ Med. 2001;58:569-574.

8. Liu Y, Tanaka H; Fukuoka Heart Study Group. Overtime work, insufficient sleep, and risk of non-fatal acute myocardial infarction in Japanese men. Occup Environ Med. 2002;59:447-451.

9. Kuwahara K, Imai T, Nishihara A, et al; Japan Epidemiology Collaboration on Occupational Health Study Group. Overtime work and prevalence of diabetes in Japanese employees: Japan epidemiology collaboration on occupational health study. PLoS One. 2014;9:e95732.

10. $\mathrm{Hu} \mathrm{H}$, Kurotani K, Sasaki N, et al; Japan Epidemiology Collaboration on Occupational Health Study Group. Optimal waist circumference cut-off points and ability of different metabolic syndrome criteria for predicting diabetes in Japanese men and women: Japan Epidemiology Collaboration on Occupational Health Study. BMC Public Health. 2016;16:220.

11. Nanri A, Nakagawa T, Kuwahara K, et al; Japan Epidemiology Collaboration on Occupational Health Study Group. Development of risk score for predicting 3-year incidence of type 2 diabetes: Japan Epidemiology Collaboration on Occupational Health Study. PLoS One. 2015;10:e0142779.

12. Kageyama T, Nishikido N, Kobayashi T, Kurokawa Y, Kabuto M. Commuting, overtime, and cardiac autonomic activity in Tokyo. Lancet. 1997;350:639.

13. Nonogaki K. New insights into sympathetic regulation of glucose and fat metabolism. Diabetologia. 2000;43:533-549.

14. Meerlo P, Sgoifo A, Suchecki D. Restricted and disrupted sleep: effects on autonomic function, neuroendocrine stress systems and stress responsivity. Sleep Med Rev. 2008;12:197-210.

15. Imai T, Kuwahara K, Miyamoto T, et al; Japan Epidemiology Collaboration on Occupational Health Study Group. Validity and reproducibility of self-reported working hours among Japanese male employees. J Occup Health. 2016;58:340-346. 